\title{
HLA loss of heterozygosity-mediated discordant responses to immune checkpoint blockade in squamous cell lung cancer with renal metastasis
}

\author{
Sheng $\mathrm{Yu}^{\ddagger, 1}$, Zhikun Zhao ${ }^{\ddagger 2}$, Longyun Chen², Tiantian $\mathrm{Gu}^{2}$, Hui Yu², Hong Tang ${ }^{1}$, Qiming \\ Wang ${ }^{* *, 1}$ \& Yufeng Wu $\mathbf{u}^{*, 1}$ \\ ${ }^{1}$ Department of Internal Medicine, Henan Cancer Hospital, Affiliated Cancer Hospital of Zhengzhou University, \\ Zhengzhou 450008, China \\ ${ }^{2}$ YuceBio Technology Co., Ltd, Shenzhen 51800, China \\ *Author for correspondence: yufengwu0dr@163.com \\ **Author for correspondence: qimingwang1006@163.com \\ $¥$ Authors contributed equally
}

Despite the significant success of immune checkpoint blockade therapy in advanced non-small-cell lung cancer compared with chemotherapy, efficacy varies greatly across patients, and acquired resistance frequently occurs. In particular, during immunotherapy, the dynamic changes in molecular events have not been characterized. The authors report a case of squamous cell lung carcinoma with renal metastasis, treated with pembrolizumab, in which the primary tumor and rare renal metastases showed different responses. Using whole-exome sequencing, the authors found loss of heterogeneity in HLA genes in all tumors and high levels of intratumor heterogeneity in metastases. The increased levels of HLA loss led to therapy resistance during tumor evolution. In addition to tumor mutational burden and PD-L1, HLA loss of heterozygosity and intratumor heterogeneity should be taken into consideration during immunotherapy.

First draft submitted: 11 June 2020; Accepted for publication: 5 November 2020; Published online:

26 November 2020

Keywords: acquired resistance $\bullet$ HLA LOH • immune checkpoint blockade $\bullet$ NSCLC

Non-small-cell lung cancer (NSCLC) is a malignant neoplasm with high incidence and mortality in China [1]. Squamous cell lung carcinoma is a common type of NSCLC. Renal metastasis has been documented in a few clinical cases [2,3]. Immune checkpoint blockade, including PD-1, PD-L1 and cytotoxic T-lymphocyte-associated antigen 4, has shown favorable outcomes in the treatment of advanced NSCLC compared with chemotherapy [4,5]. Pembrolizumab, an anti-PD-1 blockade agent, was approved by the US FDA for the treatment of patients with metastatic NSCLC in 2016.

However, only a subset of patients derive durable clinical benefit with immune checkpoint blockade, and the majority of patients eventually show resistance to therapy after an initial response. Studies on the mechanisms for the acquisition of resistance to immune checkpoint blockade remain limited. The occurrence of HLA loss of heterozygosity $(\mathrm{LOH})$, meaning the disruption of the ability to present neoantigens, is considered one pattern of immune evasion [6,7]. It has been reported that HLA LOH occurs in $40 \%$ of cases of early-stage NSCLC and more frequently than HLA or B2M mutations [6]. However, whether the presence of HLA LOH leads to resistance to immune checkpoint blockade and how to change the HLA allele-specific copy number under immune pressure remain uncertain.

In this study, the authors report the case of a treatment-naive patient with squamous cell lung carcinoma and renal metastasis who experienced complete disease remission in the primary tumor but tumor progression in the metastatic tumor after initial response to treatment with anti-PD-1 monotherapy (pembrolizumab). The authors performed a genomic analysis using paired whole-exome sequencing data to deeply explore the mechanism of acquired resistance to PD-1 blockade in metastatic tumors.

Future Medicine 


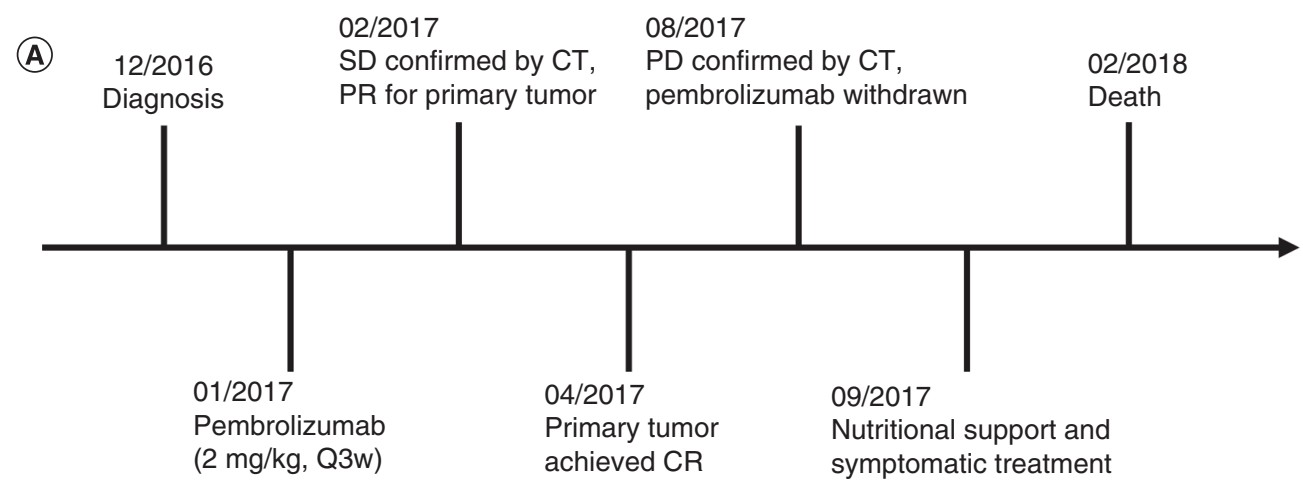

(B)
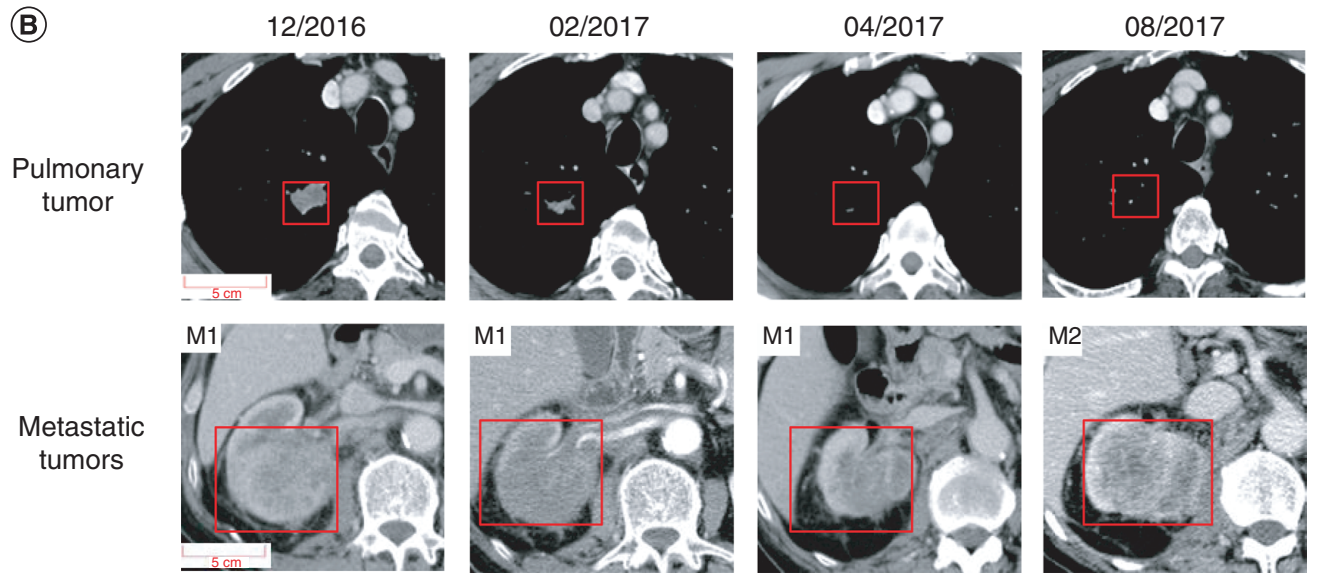

Figure 1. Clinical therapeutic responses of the patient. (A) Chronological schema of treatments. (B) CT images of the response during treatment.

CR: Complete response; CT: Computed tomography; PD: Progressive disease; PR: Partial response; Q3w: Every 3 weeks; SD: Stable disease.

\section{Case presentation}

A 75-year-old man was admitted to the hospital in December 2016 for low back pain with a numerical rating scale score of 5 . The patient was a smoker with no family history of cancer. Chest and abdominal computed tomography (CT) showed a mass in the upper lobe of the right lung $(22.1 \times 13.6 \mathrm{~mm})$ and scattered nodules and a spherical mass in the right kidney $(52.2 \times 43.9 \mathrm{~mm})$ (Figure 1). No other mass or lymph node enlargement was found. The patient received percutaneous CT-guided right lung mass biopsy and percutaneous ultrasound-guided right renal mass biopsy. Pathological analysis showed stage IV (cT4N0 M1a) squamous cell lung carcinoma (pulmonary tumor $[\mathrm{P}]$ ) and metastatic squamous cell renal carcinoma (M1) (Figure 2A \& B). Immunohistochemical staining of $\mathrm{P}$ showed positive CK7, P40, CK5/6 and TTF-1; Ki-67 index of 40\%; and negative napsin A, Syn and CD56. The serum tumor markers found included carcinoembryonic antigen at $17.33 \mathrm{ng} / \mathrm{ml}$ (normal range: $0-3.4 \mathrm{ng} / \mathrm{ml}$ ), neuron-specific enolase at $13.30 \mathrm{ng} / \mathrm{ml}$ (normal range: $0-12.5 \mathrm{ng} / \mathrm{ml}$ ) and CYFRA21-1 at $19.84 \mathrm{ng} / \mathrm{ml}$ (normal range: $0-3.3 \mathrm{ng} / \mathrm{ml}$ ). Molecular analyses of $E G F R$ and $A L K$ revealed no mutation.

The renal focus was a single metastatic focus with symptoms of low back pain. Surgical resection of renal metastases or local radiotherapy and postoperative chemotherapy may bring the best survival benefits for patients [8-10]. Clinically, the patient firmly refused surgery, radiotherapy and chemotherapy and asked for palliative treatment. Considering the small side effects of immunotherapy, long-lasting efficacy and approval by the FDA [11], the expression of PD-L1 was detected. The PD-L1 expression level was 5 and $3 \%$ in P and renal tumors (M1), respectively (Figure 2C \& D). Immunotherapy was chosen to meet the patient's wishes and improve his quality of life and survival. After a multidisciplinary panel meeting, the patient received two cycles of pembrolizumab $(2 \mathrm{mg} / \mathrm{kg}$ every 3 weeks $)$ immunotherapy from January to February 2017. The only side effect during this period was two episodes of hematuria. Symptoms of low back pain were significantly relieved, the numerical rating scale score was 2 and no painkillers were taken. At the end of February 2017, a CT scan of the chest and abdomen showed that P 
Figure 2. Pathological features of the patient. HE staining $(\times 200)$ of $(A)$ pulmonary tumor $(\mathrm{P})$ and $(\mathrm{B})$ renal metastasis (M1). PD-L1 staining $(\times 400)$ of $(C) P$ and $(D) M 1$. HE: Hematoxylin and eosin.
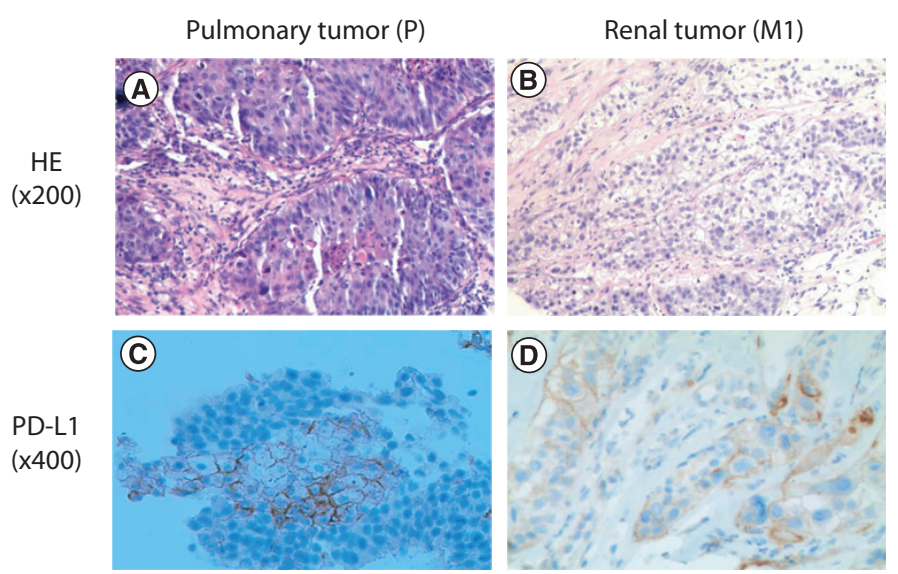

was significantly reduced in size, and the standard curative effect evaluation of RECIST 1.1 showed a partial response. M1 were slightly reduced in size, and the curative effect was evaluated as stable disease (Figure 1B). The patient continued to receive immunotherapy, and his condition was evaluated by CT scan every two cycles. P decreased gradually until it disappeared, and the best curative effect was complete response.

M1 remained stable until 30 weeks later, when tumor invaded the right psoas major muscle (Figure 1B). At the same time, symptoms of low back pain were aggravated, and the numerical rating scale pain score was 7 . It was suggested that disease progression and immunotherapy should be discontinued. To determine the next treatment plan and find the cause of drug resistance in immunotherapy, the patient underwent metastatic renal biopsy (M2) again, and the pathology suggested cancer. All three tumor biopsies, P, M1 and M2, were further sent for whole-exome sequencing as previously described [12]. The authors first compared the mutations of the three lesions and constructed a phylogenetic tree using PyClone (Figure 3A \& B) [13]. The results confirmed the tumor evolution process of $\mathrm{P}$ to $\mathrm{M} 1$ and $\mathrm{M} 1$ to M2 (Figure 3A). The tumor mutational burden (TMB) values were 6.0 muts/Mb, 15.2 muts/ $\mathrm{Mb}$ and 6.8 muts/Mb, respectively (Figure $3 \mathrm{C}$ ). No reported therapy-resistant mutations were detected. However, all lesions had LOH in HLA-I alleles HLA-A*02:06, HLA-B*51:02 and HLA-C*15:01. By inferring the clonality of HLA LOH [1], it was possible to determine that clonal LOH events occurred at the early stage of tumor evolution, which might lead to tumor initiation and immune escape (Figure 3B). In particular, M2 harbored subclonal loss in the remaining three HLA-I alleles, HLA-A*24:02, HLA-B*57:01 and HLA-C*06:02, further contributing to therapy resistance (Figure $3 \mathrm{~B}$ ). After a multidisciplinary panel meeting, pembrolizumab was withdrawn because of immune evasion. The patient still refused radiotherapy and chemotherapy and asked for symptomatic treatment. The patient was administered symptomatic treatment, including pain relief, nutritional support and psychological comfort. Unfortunately, the patient died 5 months later. To better understand the potential mechanism of therapy resistance, the authors calculated the HLA-corrected TMB [14] and intratumor heterogeneity (ITH), defined as the ratio of subclonal mutations to all mutations (Figure 3C \& D). Compared with the primary tumor, metastases M1 and M2 displayed larger decreases in HLA-corrected TMB and higher levels of ITH.

\section{Discussion}

Squamous cell carcinoma of the lung is a subtype of NSCLC that can metastasize to multiple organs. Radiotherapy and chemotherapy are the first choice for late NSCLC with negative driving genes. Renal metastasis has been documented in a few clinical cases, although it is not uncommon in clinical practice [15,16]. However, isolated metastases to the kidney are extremely rare, and there is still a lack of prospective studies. At present, the treatment of metastatic lesions in the kidney is still controversial. Previous case studies have shown that both radiotherapy and surgery have advantages $[10,15]$. This patient refused these treatments until his death because of the trauma of surgery and the toxic side effects of radiotherapy and chemotherapy. As a new treatment, immunotherapy mainly strengthens the immunity of the body to resist tumors. Compared with radiotherapy and chemotherapy, immunotherapy has fewer side effects and longer lasting benefits [17]. There is no significant difference in efficacy within the dose range of 2 to $10 \mathrm{mg} / \mathrm{kg}$, but dose will affect the incidence of adverse reactions [18]. Therefore, the authors chose the treatment of pembrolizumab $(2 \mathrm{mg} / \mathrm{kg}$ every 3 weeks). The patient had one occurrence of 
(A)

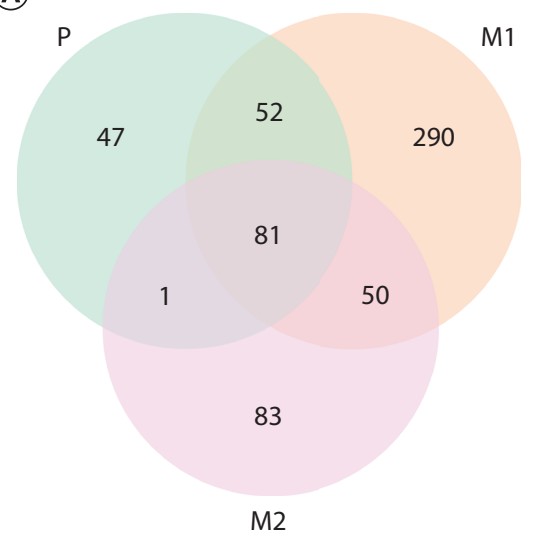

(B)

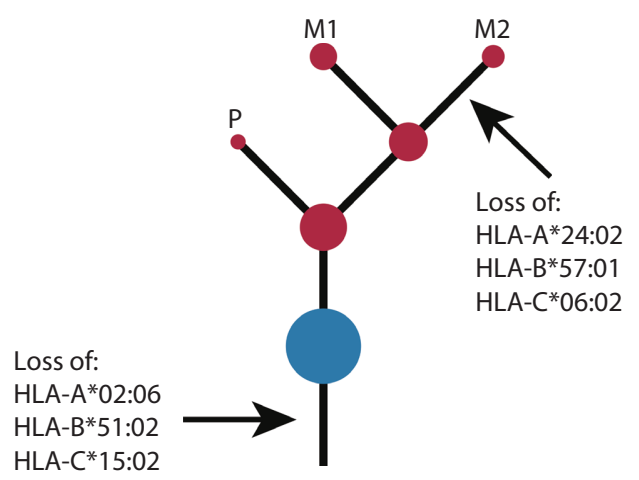

(C)



(D)

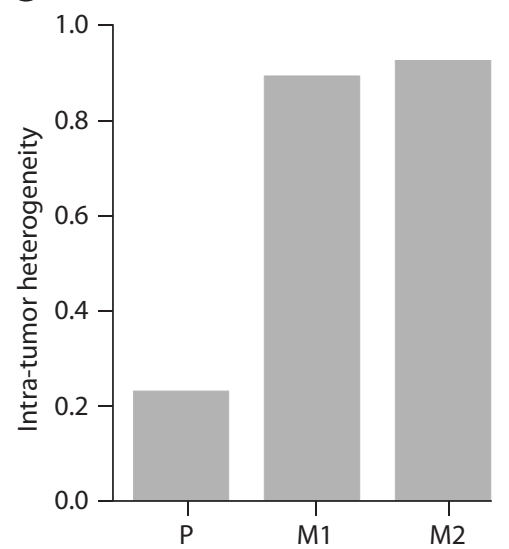

Figure 3. Genetic features of the patient. (A) Venn diagram of mutations detected in $\mathrm{P}, \mathrm{M} 1$ and $\mathrm{M} 2$. (B) Phylogenetic tree and loss of HLA genes. Clones are indicated as clonal (blue) and subclonal (red). (C) Levels of TMB of P, M1 and M2. (D) Levels of intratumor heterogeneity of $P, M 1$ and M2.

TMB: Tumor mutational burden.

hematuria after each of the first two treatments, which may have been a side effect of the drug or a therapeutic reaction, both of which were acceptable to the patient.

It has been reported that HLA LOH is a common method of immune escape, especially in lung cancer [6]. The loss of $H L A$ genes may explain why M1 with PD-L1 expression $\geq 1 \%$ and TMB of 15.2 muts/Mb did not respond to pembrolizumab monotherapy. It also demonstrated the defect of TMB as a biomarker for immunotherapy, and even monotherapy. A recent study showed that HLA-corrected TMB showed better predictive power than conventional TMB [14]. Using this method, the corrected TMB of P, M1 and M2 was 3.85 muts/Mb, 5.25 muts/Mb and 0 muts/Mb, respectively (Figure 3C). The rise of HLA LOH is usually accompanied by an increase in subclonal mutations, resulting in an increase in ITH. Increased TMB after HLA LOH might make little contribution to the response to immunotherapy because of the lack of neoantigen processing and low cancer cell fraction. Previous studies have demonstrated that low ITH is associated with a better response to immunotherapy [19-21. The ITH of the patient's three tumors showed that ITH values increased during the tumor evolution process (Figure 3D). The combination of HLA-corrected TMB and ITH of M1 might partly explain the failure of pembrolizumab monotherapy. Considering the relevance of HLA LOH and increased ITH, it is reasonable to consider the influence of ITH on TMB calculation.

There were also limitations in the authors' study. It would be informative to evaluate the tumor immune microenvironment and ITH by using anti-CD8 and/or anti-CD3 monoclonal antibodies. At the same time, increasing the staining data of HLA-I staining and B2M staining was helpful in confirming the lack of expression of HLA-I in M2. The aforementioned experiments could add to the discussion regarding ITH and the immune 
microenvironment. Unfortunately, the tumor tissue the authors obtained in each mass biopsy sample was limited. The loss of class I HLA genes may trigger the drug resistance of pembrolizumab, but it cannot rule out the influence of the immune microenvironment, T-cell function and other factors. Hence, expanded cohorts are needed to explore the influence of ITH on TMB and changes in the tumor microenvironment after HLA LOH occurs.

\section{Conclusion}

The authors have reported the case of a squamous cell lung cancer patient with discordant responses to pembrolizumab in primary and metastatic tumors. The primary tumor achieved complete response shortly after therapy, whereas the renal metastasis developed progressive disease. The results of this case analysis showed that increased levels of HLA LOH and ITH in metastasis might lead to drug resistance and failure of treatment even with high levels of TMB and expression of PD-L1. Hence, except for TMB and PD-L1, HLA LOH and ITH should be taken into consideration during immunotherapy.

\section{Future perspective}

At present, immunotherapy is widely used in first-line treatment of lung cancer, and the phenomenon of immune tolerance and immune evasion is very noteworthy during treatment. Some mechanisms of drug resistance and evasion have also been found, such as the loss of HLA heterozygosity. In this squamous cell lung carcinoma patient with rare renal metastasis, the response of the primary and metastatic lesions to immunotherapy was inconsistent. Using next-generation sequencing technology, the authors found that immune-related features, such as HLA and ITH, in the metastatic lesions were inconsistent with those in the primary lesion. The loss of HLA heterozygosity and high ITH are characteristic of immune evasion. This case suggests that immunotherapy not only needs comprehensive immune marker determination but also needs more detailed detection and evaluation for patients with multiple lesions and metastases. We still have much work to do in immunotherapy.

\section{Executive summary}

- The efficacy of immune checkpoint blockade therapy in advanced non-small-cell lung cancer varies greatly across patients, and acquired resistance frequently occurs.

- The authors report a case in which a squamous cell lung cancer patient displayed a discordant response to immunotherapy between the primary tumor (complete response) and the metastatic tumors (progressive disease).

- HLA loss of heterozygosity (LOH) occurred in the primary tumor, and the HLA genes were totally lost in the metastatic tumors after immunotherapy, indicating that HLA LOH was the potential mechanism of drug resistance.

- Tumor mutational burden increased dramatically in the metastatic tumors, accompanied by an increase in intratumor heterogeneity. Although the metastatic tumors showed tumor mutational burden-high, most mutations were subclonal and may not have contributed to the neoantigen because of HLA LOH.

- In addition to tumor mutational burden, HLA LOH and intratumor heterogeneity should be taken into consideration with immunotherapy, and biomarkers should be detected not only in primary tumors but also in metastatic tumors.

\section{Author contributions}

S Yu and Z Zhao were responsible for analyzing the data and writing the manuscript. $H$ Tang and $S$ Yu were responsible for collecting samples and clinical data as well as supervising the pathological interpretation of samples. Y Wu, Q Wang, Z Zhao, L Chen and T Gu were responsible for revising the manuscript. H Yu was responsible for submitting the manuscript. Y Wu and Q Wang were responsible for designing the study. All authors read and approved the final manuscript.

\section{Financial \& competing interests disclosure}

This manuscript was supported by the 51282 project Leading Talent of Henan Provincial Health Science and Technology Innovation Talents (grant number [2016]32), the Medical Science and Technology Program of Henan Province (grant number 201702249), the Natural Science Foundation of Henan Province (grant numbers 162300410300, 182300410297, 182300410376) and the Science and Technology Program of Henan Province (grant number 162102310327). The authors have no other relevant affiliations or financial involvement with any organization or entity with a financial interest in or financial conflict with the subject matter or materials discussed in the manuscript. This includes employment, consultancies, honoraria, stock ownership or options, expert testimony, grants or patents, received or pending, or royalties.

No writing assistance was utilized in the production of this manuscript. 
Ethical conduct of research

This study was approved by the ethics review committee of the Affiliated Cancer Hospital of Zhengzhou University (ethics review number 2019016), and written informed consent was obtained from the enrolled patient.

\section{Open access}

This work is licensed under the Attribution-NonCommercial-NoDerivatives 4.0 Unported License. To view a copy of this license, visit http://creativecommons.org/licenses/by-nc-nd/4.0/

\section{Data sharing statement}

The data analyzed for this manuscript are available from the corresponding authors on reasonable request.

\section{References}

Papers of special note have been highlighted as: $\bullet \bullet$ of considerable interest

1. Chen W, Zheng R, Baade PD et al. Cancer statistics in China, 2015. CA Cancer J. Clin. 66(2), 115-132 (2016).

2. Verma V, Simone CB 2nd. Stereotactic body radiation therapy for metastases to the kidney in patients with non-small cell lung cancer: a new treatment paradigm for durable palliation. Ann. Palliat. Med. 6(2), 96-103 (2017).

3. Cai J, Liang G, Cai Z, Yang T, Li S, Yang J. Isolated renal metastasis from squamous cell lung cancer. Multidiscip. Respir. Med. 8(1), 2 (2013).

4. Rittmeyer A, Barlesi F, Waterkamp D et al. Atezolizumab versus docetaxel in patients with previously treated non-small-cell lung cancer (OAK): a phase 3, open-label, multicentre randomised controlled trial. Lancet 389(10066), 255-265 (2017).

5. Reck M, Rodriguez-Abreu D, Robinson AG et al. Pembrolizumab versus chemotherapy for PD-L1-positive non-small-cell lung cancer. N. Engl. J. Med. 375(19), 1823-1833 (2016).

6. Mcgranahan N, Rosenthal R, Hiley CT et al. Allele-specific HLA loss and immune escape in lung cancer evolution. Cell 171(6), 1259-1271.e11 (2017).

-. Results demonstrated that HLA loss of heterozygosity $(\mathrm{LOH})$ is related to immune escape in lung cancer.

7. Chowell D, Morris LGT, Grigg CM et al. Patient HLA class I genotype influences cancer response to checkpoint blockade immunotherapy. Science 359(6375), 582-587 (2018).

-. Results demonstrated that HLA class I genotype influences the clinical efficacy of PD-L1/PD-1 inhibitors.

8. Walther MM, Alexander RB, Weiss GH et al. Cytoreductive surgery prior to interleukin-2-based therapy in patients with metastatic renal cell carcinoma. Urology 42(3), 250-257 (1993).

9. Dabestani S, Marconi L, Hofmann F et al. Local treatments for metastases of renal cell carcinoma: a systematic review. Lancet Oncol. 15(12), e549-e561 (2014)

10. Adamy A, Von Bodman C, Ghoneim T, Favaretto RL, Bernstein M, Russo P. Solitary, isolated metastatic disease to the kidney: Memorial Sloan-Kettering Cancer Center experience. BJU Int. 108(3), 338-342 (2011).

11. Morgensztern D, Herbst RS. Nivolumab and pembrolizumab for non-small cell lung cancer. Clin. Cancer Res. 22(15), 3713-3717 (2016).

12. Wu D, Liu Y, Li X et al. Identification of clonal neoantigens derived from driver mutations in an EGFR-mutated lung cancer patient benefitting from anti-PD-1. Front. Immunol. 11, 1366 (2020).

13. Roth A, Khattra J, Yap D et al. PyClone: statistical inference of clonal population structure in cancer. Nat. Methods 11(4), 396-398 (2014).

14. Shim JH, Kim HS, Cha $\mathrm{H}$ et al. HLA-corrected tumor mutation burden and homologous recombination deficiency for the prediction of response to PD-(L)1 blockade in advanced non-small-cell lung cancer patients. Ann. Oncol. 31(7), 902-911 (2020).

15. Verma V, Simone CB 2nd. Stereotactic body radiation therapy for metastases to the kidney in patients with non-small cell lung cancer: a new treatment paradigm for durable palliation. Ann. Palliat. Med. 6(2), 96-103 (2017).

16. Wang J, Wang L, Long L, Tao Q, Xu F, Luo F. Solitary renal metastasis from squamous cell carcinoma of the lung: a case report. Medicine (Baltimore) 98(5), e14310 (2019).

17. Herbst RS, Baas P, Kim DW et al. Pembrolizumab versus docetaxel for previously treated, PD-L1-positive, advanced non-small-cell lung cancer (KEYNOTE-010): a randomised controlled trial. Lancet 387(10027), 1540-1550 (2016).

18. Chatterjee M, Turner DC, Felip E et al. Systematic evaluation of pembrolizumab dosing in patients with advanced non-small-cell lung cancer. Ann. Oncol. 27(7), 1291-1298 (2016).

19. Zhang J, Fujimoto J, Zhang J et al. Intratumor heterogeneity in localized lung adenocarcinomas delineated by multiregion sequencing. Science. 346(6206), 256-259 (2014).

20. Landau DA, Carter SL, Stojanov P et al. Evolution and impact of subclonal mutations in chronic lymphocytic leukemia. Cell. 152(4), 714-726 (2013).

21. Sutherland KD, Visvader JE, Cellular mechanisms underlying intertumoral heterogeneity. Trends. Cancer. 1(1), 15-23 (2015). 